\title{
Microsurgical fenestration of perineural cysts to the thecal sac at the level of the distal dural sleeve
}

\author{
Axel Neulen • Sven R. Kantelhardt • \\ Sara M. Pilgram-Pastor • Imke Metz • Veit Rohde • \\ Alf Giese
}

Received: 23 February 2011 / Accepted: 26 April 2011 / Published online: 12 May 2011

(C) The Author(s) 2011. This article is published with open access at Springerlink.com

\begin{abstract}
Background Surgery for symptomatic sacral perineural cysts remains an issue of discussion. Assuming microcommunications between the cyst and thecal sac resulting in a valve mechanism and trapping of CSF as a pathomechanism, microsurgical fenestration from the cyst to the thecal sac was performed to achieve free CSF communication.

Methods In 13 consecutive patients (10 female, 3 male), MRI revealed sacral perineural cysts and excluded other pathologies. Micro-communication between the thecal sac and the cysts was shown by delayed contrast filling of the cysts on postmyelographic CT. Surgical fenestration achieved free CSF communication between the thecal sac and cysts in all patients. The patient histories, follow-up
\end{abstract}

Parts of this study were presented at the 61st Annual Meeting of the German Society of Neurosurgery (DGNC) in 2010.

A. Neulen · S. R. Kantelhardt $\cdot$ S. M. Pilgram-Pastor • V. Rohde ·

A. Giese

Department of Neurosurgery, Center of Neurological Medicine,

University of Göttingen,

Göttingen, Germany

S. M. Pilgram-Pastor

Department of Neuroradiology,

Center of Neurological Medicine, University of Göttingen,

Göttingen, Germany

I. Metz

Department of Neuropathology, Center of Neurological Medicine, University of Göttingen,

Göttingen, Germany

A. Neulen $(\bowtie) \cdot$ S. R. Kantelhardt $\cdot$ A. Giese

Department of Neurosurgery,

Johannes Gutenberg University of Mainz,

Langenbeckstrasse 1,

55131 Mainz, Germany

e-mail: axel.neulen@gmx.de examinations and self-assessment scales were analyzed. Symptoms at initial presentation included lumbosacral pain, pseudoradicular symptoms, genital pain and urinary dysfunction. Mean follow-up was 10.7 \pm 6.6 months.

Findings Besides one CSF fistula, no surgical complications were observed. Five patients did not improve after surgery; in four of these cases multiple cysts were found, but small and promptly filling cysts remained untreated. Seven patients reported lasting benefit following surgery; three of these had single cysts, and all had cysts $>1 \mathrm{~cm}$. One patient initially benefited from cyst fenestration but experienced recurrent pain within 2 months postoperatively. Re-myelography revealed delayed contrast filling of the recurrent cyst; however, surgical revision did not lead to an improvement despite successful fenestration and collapse of the cyst revealed by postoperative imaging.

Conclusions Microsurgical fenestration of sacral perineural cysts to the thecal sac is a surgical approach that has shown success in the treatment of lumbosacral pain, pseudoradicular symptoms, genital pain and urinary dysfunction associated with sacral perineural cysts. Our analysis, however, shows that mainly patients with singular large cysts benefit from this treatment.

Keywords Sacral perineural cyst · Tarlov cyst - Meningeal cyst $\cdot$ Meningeal diverticulum $\cdot$ Sacral radiculopathy

\section{Introduction}

Sacral perineural cysts (Tarlov cysts) are cysts of the sacral nerve roots. They rarely become symptomatic, but have been associated with lumbar and sacral pain, pseudoradicular and radicular symptoms, and bowel and bladder dysfunction $[1-5,7,10,11]$. Perineural cysts, most of 
which were asymptomatic, showed a prevalence of $1.5 \%$ to $4.6 \%$ in radiological studies $[6,9]$. Their etiology remains unclear (reviewed in [5]). Micro-communications at the distal dural sleeve of the nerve root functioning as a valve that allows CSF influx while restricting CSF efflux is thought to be one possible underlying pathomechanism of perineural cyst growth [8]. Cyst expansion may eventually lead to irritation or compression of the affected or adjacent nerve roots, and erosion of surrounding bone with consecutive irritation of periostal pain fibers.

Cystic lesions of the sacral region can be diagnosed by $\mathrm{CT}$ or MRI. The presence of a communication between the cyst and thecal sac differentiates perineural cysts from other cystic lesions [8]. These micro-communications can be demonstrated by myelography and postmyelographic CT. Delayed contrast filling of the cystic lesion indicates the presence of micro-communications and hence a possible valve mechanism $[1,2,7,8,11]$.

Surgical management of sacral perineural cysts remains a matter of discussion. Numerous strategies have been proposed, including cyst fenestration, cyst imbrication, cyst resection, neck ligation and percutaneous aspiration $[1-5,7$, $10,11]$. Based on the supposed pathomechanism, we used a microsurgical approach for symptomatic sacral perineural cysts that establishes a free communication between the thecal sac and the perineural cyst. In this study we report our experience with 13 patients who underwent surgery for sacral perineural cysts between June 2008 and October 2010.

\section{Patients and methods}

\section{Patients}

The study was approved by the ethics committee of the medical faculty of the University of Göttingen, protocol no. 23/7/09. Patients who presented with lumbosacral pain, pseudoradicular and radicular symptoms, genital pain or urinary dysfunction were examined by MRI and routine neurological examination. If cystic lesions of the sacral region were diagnosed, other reasons for patients' symptoms were excluded. If conservative treatment failed to relieve symptoms, myelography with early and delayed postmyelographic $\mathrm{CT}$ was performed.

The early postmyelographic plain x-rays and CT scans were obtained directly after contrast application; late postmyelographic CT was done $3-4 \mathrm{~h}$ after contrast application. Delayed filling of cysts becomes apparent as lack or partial contrast filling of the cyst in the early postmyelographic $\mathrm{CT}$ and increased contrast filling in the late postmyelographic $\mathrm{CT}$, which can be verified by an increase in Hounsfield units (Fig. 1). Patients with promptly
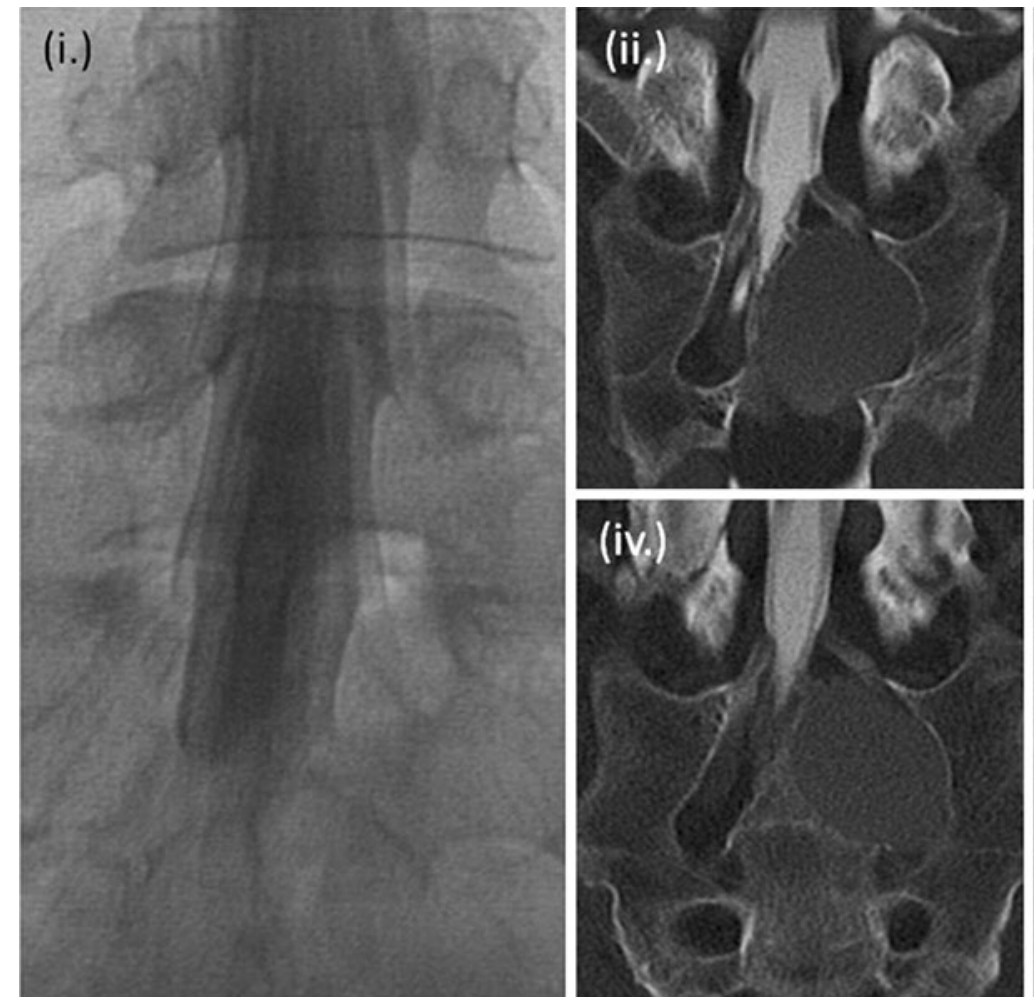
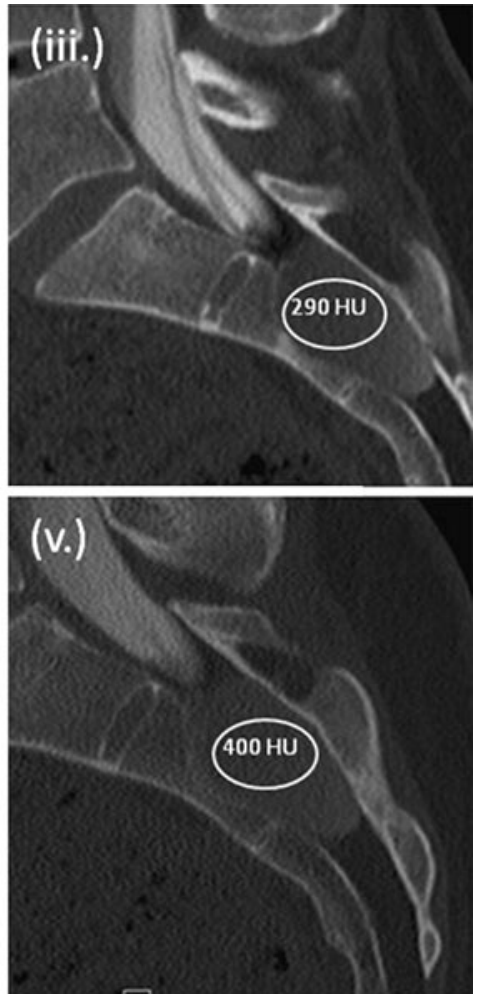

Fig. 1 Imaging findings. Anteroposterior myelography (i), coronal (ii, iv) and sagittal (iii, v) postmyelographic CT reformations demonstrated a delayed contrast filling of the cysts in all patients considered for surgery (ii and iii: early postmyelographic CT; iv and v: late postmyelographic CT). Numbers indicate a ROI analysis for Hounsfield units (HU) 
contrast enhancing lesions in myelography and early postmyelographic CT were not scheduled for surgery, while delayed contrast filling indicated the presence of microcommunications and a possible valve mechanism. In these patients surgery was considered. Ten patients had two or more cysts; in seven of these only the largest cysts thought to be responsible for the patient's symptoms were treated, while small and promptly filling cysts were not operated on.

\section{Microsurgical technique}

Patients were operated on in the prone position under general anesthesia. A 4-cm skin incision was placed directly over the lesion (usually between L5 to S3). The sacrum was exposed, and unroofing of the sacral canal and dorsal aspects of the neuroforamen exposed the cyst. The thecal sac was exposed at the level of S1. Care was taken to preserve the integrity of the cyst wall, and dissection of the cyst from surrounding structures was limited to what was essential to expose the neck of the cyst and identify the dural sleeve of the involved nerve root (Fig. 2, i-ii). The dura of the thecal sac was opened, and CSF was drained; during CSF drainage the cyst remained filled in all cases (Fig. 2, iii), proving a restricted CSF communication suggestive of the proposed valve mechanism. The course of the involved nerve root into the dural sleeve was identified, and the dural incision was extended into the dural sleeve of the nerve root under direct microscopic visualization of the nerve (Fig. 2, iv-vi). The dural incision was continued distally and the nerve was followed into the cyst, which led to collapse of the cyst. Typically the nerve root thinned at the entry into the cyst and became part of the ventral and medio-dorsal cyst wall (Fig. 2, vii). At the termination of the dural sleeve, fibrous arachnoidal tissue was typically found to be circular and adherent to the nerve root, obstructing the neck of the cyst. The fibrous tissue was resected, the nerve root was entirely freed, and a communication through the fibrous barrier between the cyst and thecal sac was established (Fig. 2, vii). A biopsy was taken from translucent parts of the cyst wall where possible. The cyst wall was mobilized, and plication of the cyst was performed. The dural incision and the cyst wall were closed using 6.0 non-resorbable sutures; if indicated, duraplasty was performed at the neck of the dural sleeve of the nerve root. At the end of surgery free macrocommunication between the cyst and thecal sac was established in all cases (Fig. 2, viii). After dura closure lumbar drains were not required. Patient mobilization started on postoperative day 1.

\section{Neurological outcome and pain assessment}

A retrospective analysis of the patients' history was performed by review of the patient charts. Additional information was gathered from self-assessment scales and telephone interviews. Assessment included (1) preoperative symptoms, (2) number, size and location of cysts, and (3) improvement of symptoms. Symptoms were summarized in the following categories: (1) lumbosacral pain, (2) pain radiating into the buttocks, (3) pain radiating into the legs, (4) genital pain, (5) bowel/bladder dysfunction, and (6) pain radiating into the groin and abdomen.
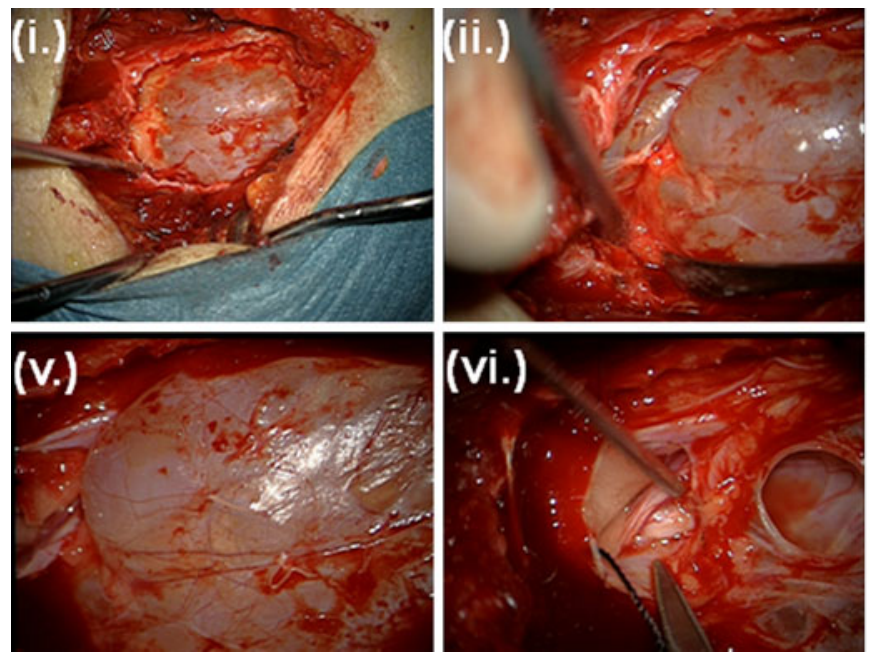

Fig. 2 Surgical approach. A sacral laminectomy was performed (i), and the thecal sac and the cyst were exposed (ii). The thecal sac was opened (iii), and the nerve was followed intradurally through the dural sleeve of the nerve root into the cyst (iv). Because of a valve mechanism the cysts did not collapse even though the thecal sac was
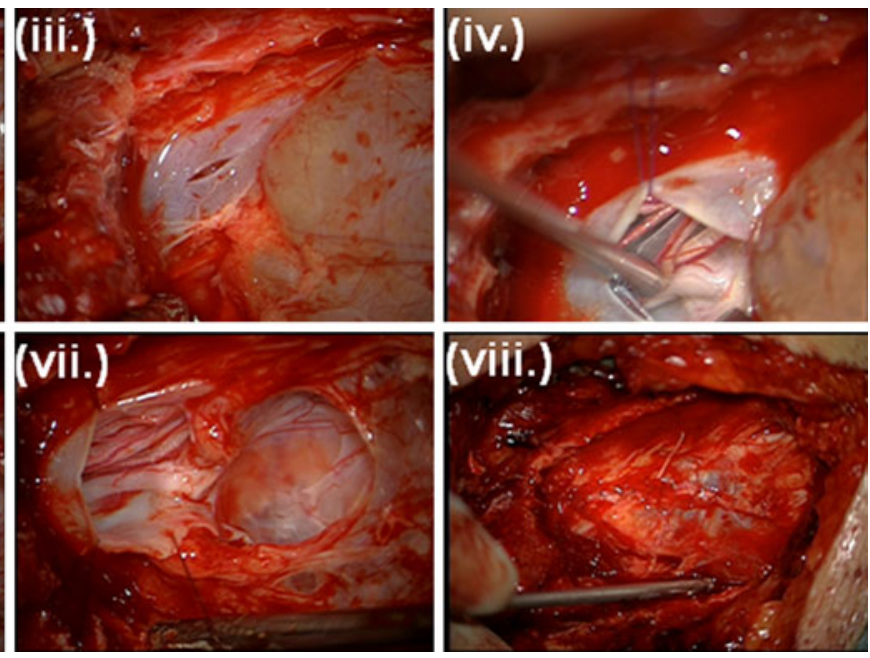

opened (v). Fibrous arachnoidal tissue obstructing the neck of the cyst was resected, establishing a communication between the cyst and the thecal sac (vi, vii). The dura was then closed with watertight sutures. Where feasible, plication of the cyst was performed (viii) 
Histopathological examination

Specimens were fixed in $4 \%$ paraformaldehyde and embedded in paraffin; 4- $\mu \mathrm{m}$-thick slices were stained with hematoxylin and eosin (HE), elastica van Gieson stain and periodic acid-Schiff stain (PAS). Immunohistochemical staining for neurofilaments was performed with a biotinavidin technique. Anti-NF antibody was used as primary antibody (anti-NF200, Sigma-Aldrich, USA).

\section{Results}

Epidemiology and clinical characteristics

Between June 2008 and October 2010, a total of 1,873 operations were performed for various spinal disorders. Thirteen patients during this period were treated for symptomatic perineural cysts and were included in this study. Mean age at surgery was $60 \pm 9.8$ years. Ten patients were female and three male. Symptoms had been present for $8.7 \pm 11.5$ years (6 months to 30 years). Postoperative mean follow-up was $10.7 \pm 6.6$ months ( 2.5 to 20 months). All perineural cysts originated from the nerve roots S1, S2 or S3. Lumbosacral pain was the most frequent symptom (10 of 13), followed by pain radiating into the buttocks ( 8 of 13), legs (7 of 13), groin and abdomen (4 of 13), genital pain (3 of 13) and bowel/bladder dysfunction (3 of 13). Figure 3 gives an overview of symptoms. No patient had a history of trauma or infection.

\section{Clinical course}

Five patients did not improve, while eight patients significantly benefited from surgery. Mostly, patients were discharged from the hospital on postoperative day 10 (5 to 14 days). One patient had to be treated with a lumbar drain for 7 days because of CSF fistula. No other complications were observed. One of the patients who initially improved experienced recurrent pain after 2 months. Re-myelography and postmyelographic CT again showed delayed postmyelographic contrast filling of a recurrent cyst; however, the patient did not benefit from reoperation, despite successful refenestration and duraplasty resulting in complete collapse and disappearance of the cyst demonstrated by postoperative imaging.

\section{Analysis of outcome}

All five patients who did not improve after surgery had multiple cysts, and in four of these patients one or several small or promptly filling cysts remained untreated; in two of the patients who did not improve, the operated cysts were smaller than $1 \mathrm{~cm}$ in diameter.
Of the eight patients who benefited from surgery, three had single cysts (among these was the patient with recurrent symptoms and reoperation). Two patients had two cysts with delayed contrast filling of similar size each. In both patients both cysts were treated. Three patients had multiple cysts, and only large and delayed filling cysts were operated on. In all patients who improved, the operated cysts were $>1 \mathrm{~cm}$ in diameter $(1.2 \mathrm{~cm}$ to $3 \mathrm{~cm})$.

Of eight patients who answered to a questioner whether surgery was successful, four responded with "no" and four with "yes." Of those who responded with "no," three had two cysts and only the larger cyst had been operated on. The remaining patient was the one with a single cyst who suffered a relapse of pain after an initial pain-free period. Of the four patients who responded with "yes," two had been treated for single cysts, and two others had two cysts and both cysts had been operated on.

Improvement related to the different symptoms was analyzed. The best improvement was documented for pain radiating into the legs and buttocks, genital pain and lumbosacral pain while improvement of bowel/bladder dysfunction and pain radiating into the groin and abdomen was observed less frequently (Fig. 4).

\section{Histological results}

HE staining showed the cyst wall with membranous tissue (Fig. 5, i). Similar to other studies, nerve fibers embedded into connective tissue were detected (Fig. 5, ii, Elastica van Gieson stain for connective tissue and Fig. 5, iii, anti-NF staining for nerve fibers). There was no evidence of inflammation.

\section{Discussion}

This report summarizes the experience from 13 patients who underwent surgical treatment for sacral perineural cysts in a single neurosurgical department between 2008 and 2010. The epidemiological characteristics of the patients in our study are similar to those reported in other series [2,5]. This is one of the largest series reported so far; to our knowledge, only one larger series of 15 patients has been presented previously [2]. However, in the latter study an approach based on cyst resection was used.

The etiology of sacral perineural cysts remains unclear [5]. However, there is general acceptance that enlargement of the cysts is caused by pulsatile and hydrodynamic forces of CSF entering the cyst combined with a valve mechanism that only allows influx of CSF $[1,2,5,7,8$, 
Fig. 3 Patient characteristicsrelative frequency of different symptoms prior to surgery. Symptoms were categorized as lumbosacral pain, pain radiating into the buttocks, legs, groin and abdomen, genital pain and bowel/bladder dysfunction

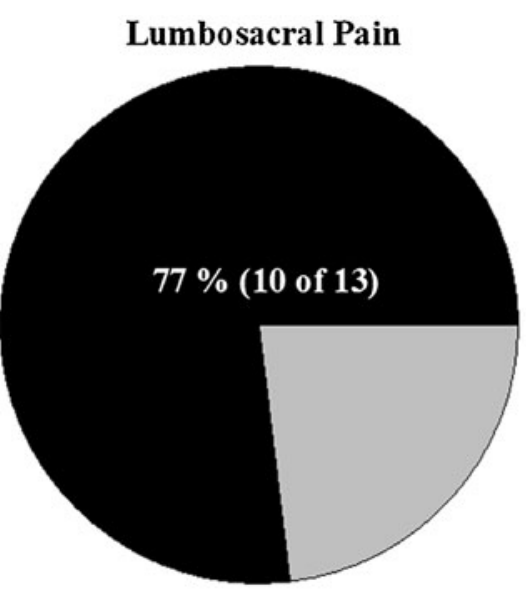

Pain radiating into the Buttocks

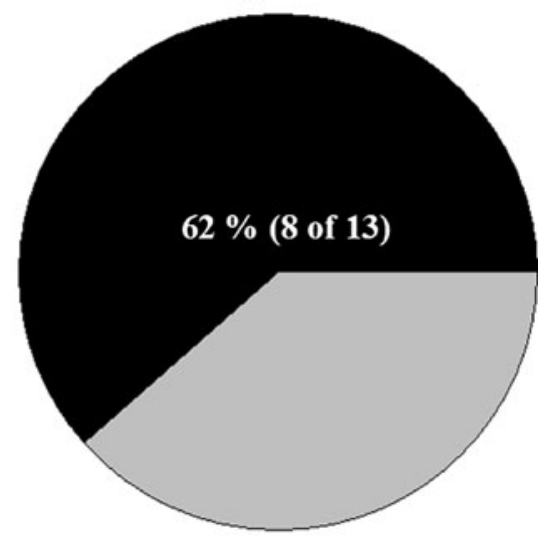

Pain radiating into Groins / Abdomen

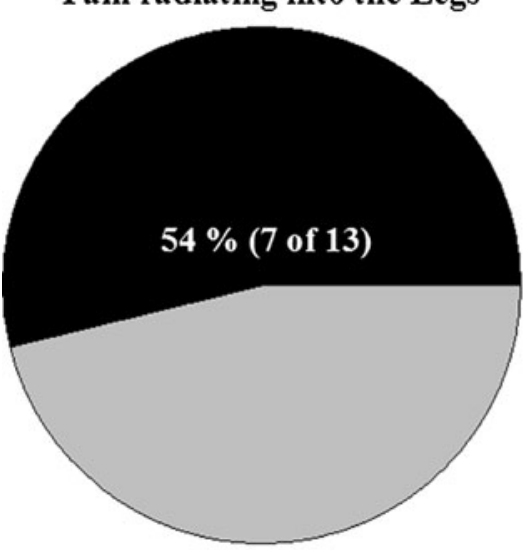

Genital Pain

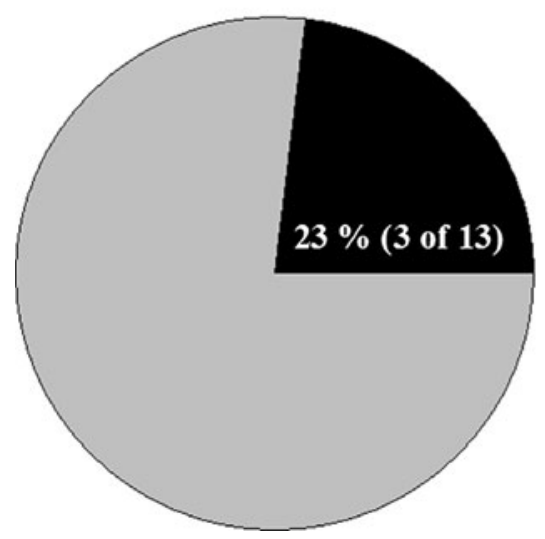

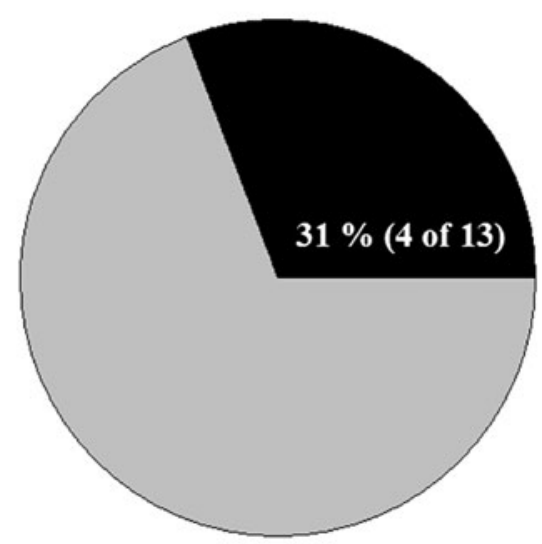

Bowel / Bladder Disturbance

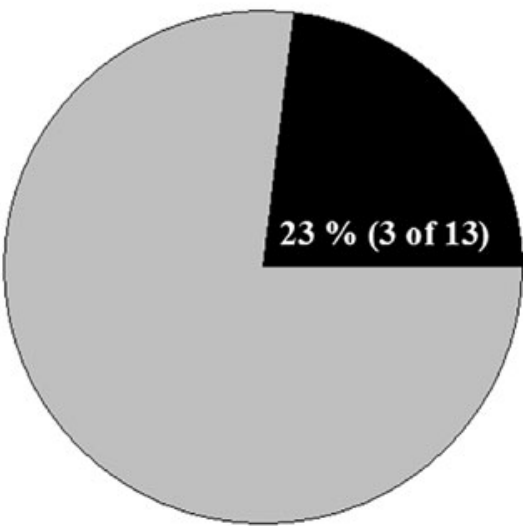

10, 11]. Similar to other studies $[1,2,7,11]$, the diagnosis of a cystic lesion of the sacral area was followed by myelography with early and late postmyelographic CT. This invasive imaging modality allows diagnosis of communications between the cyst and thecal sac, which is suspected to function as a valve. Delayed filling after intrathecal contrast application is therefore typical; this phenomenon was demonstrated by the delayed increase in Hounsfield units within the cyst compared to the thecal sac
(Fig. 1) and was the main criteria for patient selection. The valve mechanism was further confirmed by our intraoperative findings. All cysts did not collapse after incision of the thecal sac and CSF drainage, but remained filled until the cyst itself was opened (Fig. 2, iii-v).

The symptoms associated with perineural cysts can be attributed to (1) radicular involvement, i.e., sacral ischialgia and bowel/bladder dysfunction. Radicular symptoms may either result from compression of neighboring nerve roots 
Fig. 4 Patient improvement rate for different symptoms (Fig. 3)
Pain (Legs) - Improvement

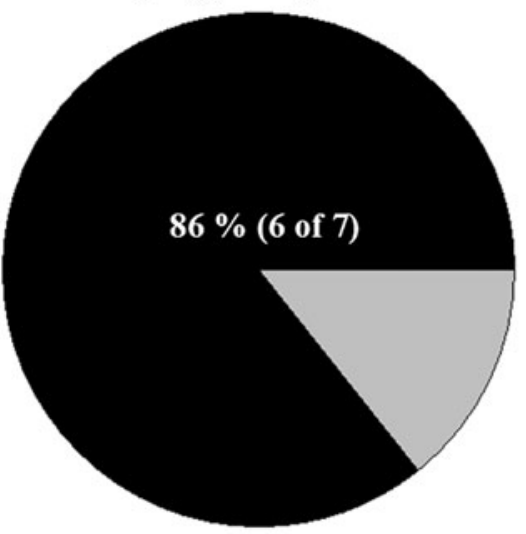

Genital Pain - Improvement

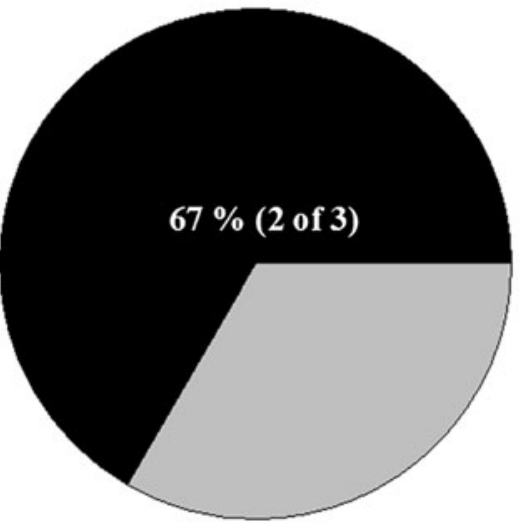

Bowel / Bladder Dysfunction Improvement

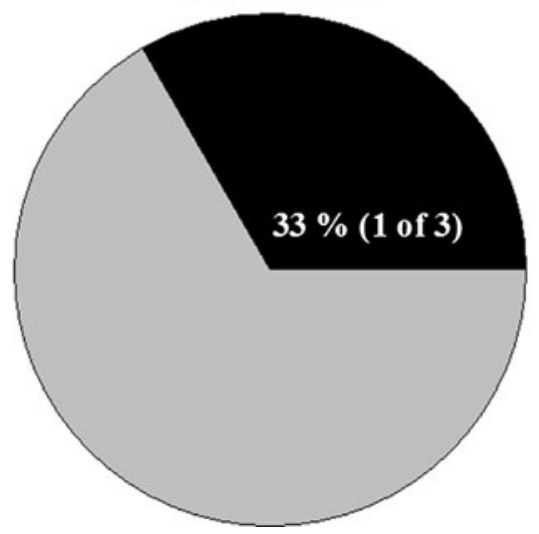

Pain (Buttocks) - Improvement

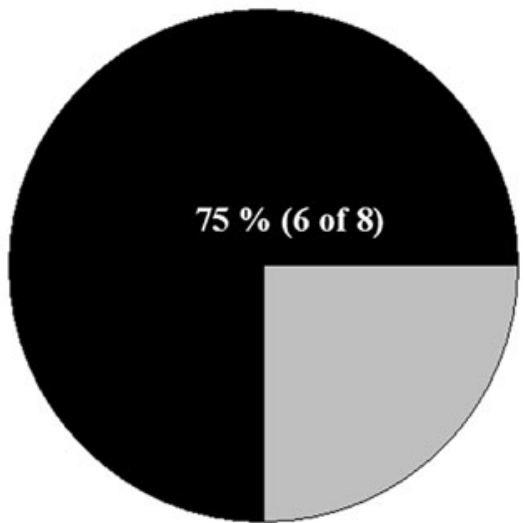

Lumbosacral Pain - Improvement

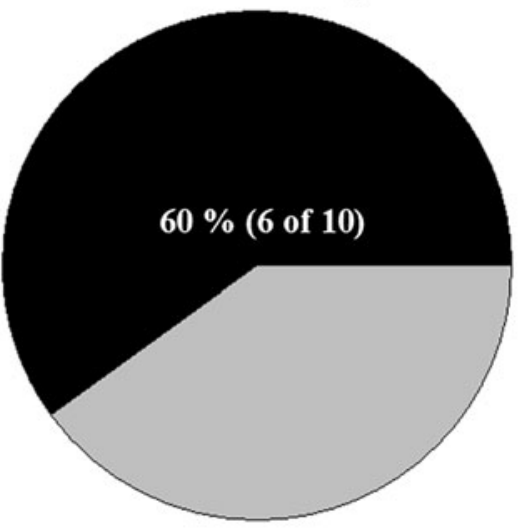

Pain radiating into Groins / Abdomen - Improvement

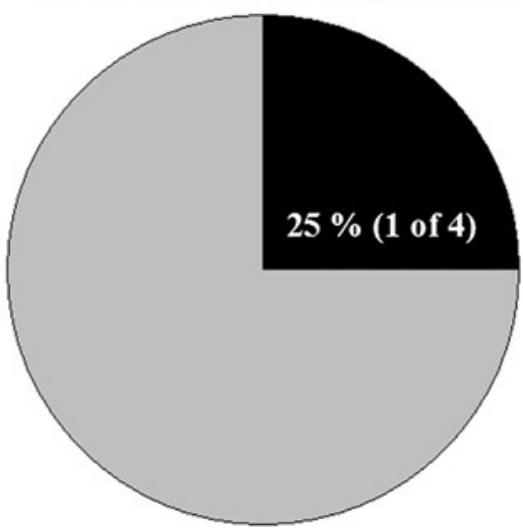

or from direct impairment of the affected nerve root itself. Further explanations for symptoms are (2) bone erosion and compression of periostal pain fibers, which may result in sacral pain, and (3) pseudoradicular radiation of pain into the buttocks, legs and groin. Our results imply that symptoms that can be attributed to bone erosion and pseudoradicular radiation are more likely to benefit from surgery than radicular symptoms (Fig. 4). The latter may be influenced by permanent impairment of the nerve itself because of structural changes of the nervous tissue as a consequence of compression or stretching by the expanding cyst, which may result in chronic deafferentiation pain.

Different surgical approaches have been used to treat perineural cysts. Most approaches are based on resection or fenestration of the cyst wall into the epidural space $[2,10$, 11], which may be followed by closure of the defect with muscle flap, gel foam or fibrin glue $[1,5]$, or neck ligation 

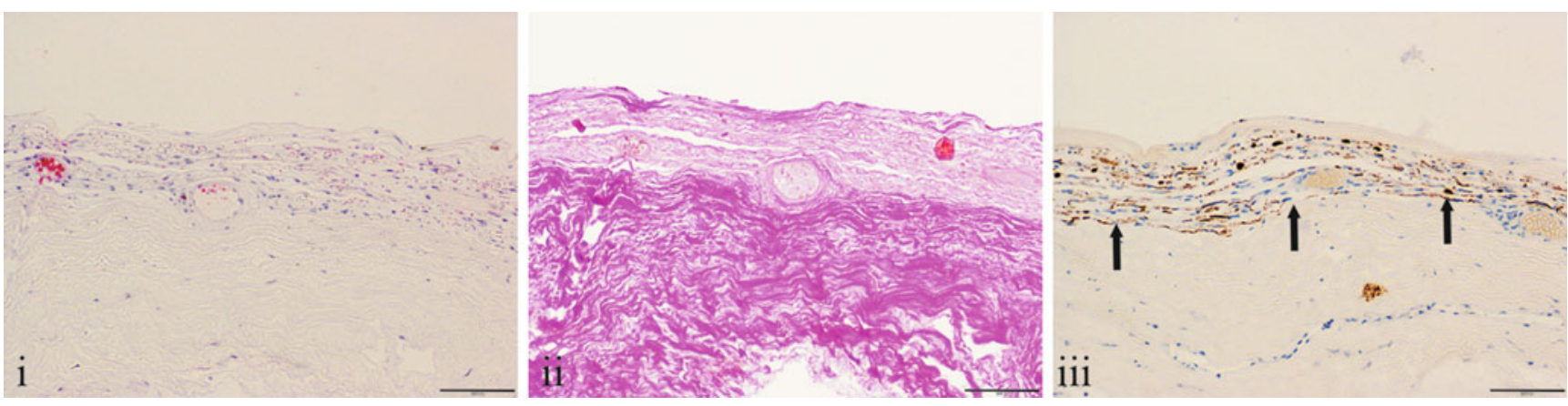

Fig. 5 Histology revealed membranous connective tissue: (i) HE staining and (ii) Elastica van Gieson stain. Nerve fibres were superficially embedded within the connective tissue [marked by arrows in (iii), anti-NF antibody]. Original magnification $(\mathrm{OM}): \times 100$. Scale bar: $500 \mu \mathrm{m}$

after cyst wall resection [7]. The authors of the latter study used image-guided percutaneous aspiration of cyst contents, which induced a collapse of the cyst and hence reduced symptoms, as a diagnostic tool prior to surgery [7]. One study reports a case of cyst fenestration and intraoperative drainage of fluid [3]; furthermore, shunting of the sacral cyst to the peritoneum has been proposed [4]. In contrast, our approach aims to establish free CSF communication between the thecal sac and cyst in order to eliminate the valve mechanism rather than resection or disruption of the cyst wall. To the best of our knowledge, this is the first study reporting a patient series using this approach despite the fact that this microsurgical approach is also used by other neurosurgical centers.

In 8 of 13 patients symptoms improved significantly, while 5 patients did not benefit from the procedure. Careful review of imaging and clinical data revealed that in our collective, patients with small cysts $(<1 \mathrm{~cm})$ did not benefit from surgery. This finding corresponds to the results of Voyadzis and co-workers who observed a correlation between cyst size and clinical outcome [11]. Therefore, we suggest that cysts of a $<1 \mathrm{~cm}$ diametershould be approached with caution even in the presence of delayed contrast filling on postmyelographic CTs. Further, we observed that in our collective all patients who did not benefit from surgery had multiple cysts, and in four of these patients small or promptly contrast-enhancing cysts remained untreated. In contrast, only three of the eight patients who improved after surgery had multiple cysts, of which, however, like in the no-benefit group, small and not promptly contrastenhancing cysts were not treated surgically. The other five patients who benefited from surgery had either large single cysts or multiple cysts of similar size, which were all treated surgically.

Therefore, we propose that:

1. In cases with multiple delayed filling cysts of similar size, all cysts should be treated surgically. We did not observe any difference in clinical outcome for patients with single or multiple large $(>1 \mathrm{~cm})$ cysts with documented delayed contrast filling on postmyelographic CT scans, if all cysts were treated surgically.

2. If multiple cysts are present, of which one or several cysts have a diameter of $<1 \mathrm{~cm}$ or one or several cysts show prompt postmyelographic contrast filling, patients should be carefully and critically informed about the risk of residual symptoms, because in our collective five of eight patients with multiple cysts of this type did not significantly benefit from surgery.

Finally, these data have to be interpreted with caution because of the relatively small size of our patient collective and the heterogenic nature of single or multiple cysts of various sizes, which may have prognostic significance as suggested by our study. Furthermore, this study is a retrospective case collection with its inherent limitations. However, all other reported series of this rarely treated entity are small and have reported various treatment strategies, which makes final recommendations on surgical strategies difficult. A prospective study will be needed to further investigate the treatment approach suggested by the present study.

\section{Conclusion}

Microsurgical fenestration between perineural cysts and the thecal sac is a safe surgical approach in the treatment of symptomatic cysts yielding results comparable to patient series with other surgical strategies $[5,11]$. Major complications are rare: 1 case of CSF leakage that was treated successfully by a lumbar drain for 7 days in a series of 13 patients. Our data suggest that patients with single or multiple perineural cysts of $>1 \mathrm{~cm}$ in diameter and delayed contrast filling on postmyelographic CTs benefit from surgery, but all of these lesions have to be treated surgically. Patients with cysts $<1 \mathrm{~cm}$ in diameter did not significantly benefit from surgery. If multiple lesions were present, of 
which some were smaller than $1 \mathrm{~cm}$ or promptly filling with contrast on postmyelographic CTs, patients had a significant risk of remaining symptomatic.

\section{Conflicts of interest None.}

Open Access This article is distributed under the terms of the Creative Commons Attribution Noncommercial License which permits any noncommercial use, distribution, and reproduction in any medium, provided the original author(s) and source are credited.

\section{References}

1. Acosta FL Jr, Quinones-Hinojosa A, Schmidt MH, Weinstein PR (2003) Diagnosis and management of sacral Tarlov cysts. Case report and review of the literature. Neurosurg Focus 15:E15

2. Caspar W, Papavero L, Nabhan A, Loew C, Ahlhelm F (2003) Microsurgical excision of symptomatic sacral perineurial cysts: a study of 15 cases. Surg Neurol 59:101-105

3. Chaiyabud P, Suwanpratheep K (2006) Symptomatic Tarlov cyst: report and review. J Med Assoc Thai 89:1047-1050

4. Guest JD, Silbert L, Casas CE (2005) Use of percutaneous endoscopy to place syringopleural or cystoperitoneal cerebrospinal fluid shunts: technical note. J Neurosurg Spine 2:498-504

5. Guo D, Shu K, Chen R, Ke C, Zhu Y, Lei T (2007) Microsurgical treatment of symptomatic sacral perineurial cysts. Neurosurgery 60:1059-1065

6. Langdown AJ, Grundy JR, Birch NC (2005) The clinical relevance of Tarlov cysts. J Spinal Disord Tech 18:29-33

7. Lee JY, Impekoven P, Stenzel W, Löhr M, Ernestus RI, Klug N (2004) CT-guided percutaneous aspiration of Tarlov cyst as a useful diagnostic procedure prior to operative intervention. Acta Neurochir (Wien) 146:667-670

8. Nabors MW, Pait TG, Byrd EB, Karim NO, Davis OD, Kobrine AI, Rizzoli HV (1988) Updated assessment and current classification of spinal meningeal cysts. J Neurosurg 68:366-377

9. Paulsen RD, Call GA, Murtagh FR (1994) Prevalence and percutaneous drainage of cysts of the sacral nerve root sheath (Tarlov cysts). AJNR Am J Neuroradiol 15:293-297

10. Tanaka M, Nakahara S, Ito Y, Nakanishi K, Sugimoto Y, Ikuma H, Ozaki T (2006) Surgical results of sacral perineural (Tarlov) cysts. Acta Med Okayama 60:65-70
11. Voyadzis JM, Bhargava P, Henderson FC (2001) Tarlov cysts: a study of 10 cases with review of the literature. J Neurosurg 95:25-32

\section{Comment}

This is an interesting article. It touches on a subject for which a clear decision-making process still eludes us: Which sacral neural cysts are really symptomatic and therefore deserve surgical treatment, and if so, what should the operation consist of?

In a period of 3 years a total of 13 patients were operated on for 'symptomatic' sacral neural cysts.

As stated by the authors, this number, albeit not very large, from a merely statistical standpoint certainly is clinically considerable. Despite the fact that these cysts are diagnosed with relative frequency, it is often difficult to understand what type of causal relationship if any they have with the patient's complaints.

The authors have carefully imaged these cysts preoperatively, paying close attention to what they think is the main feature contributing to symptoms, that is, the ball and valve communication between the cal sac and the cyst.

The outcome was not great. Five+one patient did not benefit from surgery, which amounts to virtually $50 \%$ bad results.

The authors argue that the rest of the patient population clearly benefited in terms of symptom relief, and they are convinced that they have isolated the factors associated with non-response and make their recommendations based on this assumption.

As stated, there are some caveats to the series. In statistical terms it is too small to draw any evidence from it, and even if we did the number of non-respondents to surgery certainly would advise thinking twice before recommending it. The fact that it is retrospective and that it does not have any control population with alternative treatment to match it with is of consideration, especially with this clinical entity where it is so difficult to really get a grip and understand what is causing what in terms of symptoms (as signs are so infrequent). In this sense it is always useful to include some form of patient selfassessment asking how they rated their pre- and postoperative condition and whether they would go back for the procedure once more knowing that these were the expected results.

The authors are encouraged to pursue their clinical investigation in a prospective manner and to come back to us hopefully in the near future with more patients for whom they have applied their final recommendations.

Manuel Cunha e Sa

Almada, Portugal 